\title{
From free algebras to proof bounds
}

\author{
Silvio Ghilardi ${ }^{1}$ \\ Department of Mathematics, \\ Università degli Studi, \\ Milano, Italy \\ silvio.ghilardi@unimi.it
}

\begin{abstract}
(This is joint work with Nick Bezhanishvili) [1, 2]. In the first part of our contribution, we review and compare existing constructions of finitely generated free algebras in modal logic focusing on step-by-step methods. We discuss the notions of step algebras and step frames arising from recent investigations [3, [2], as well as the role played by finite duality. A step frame is a two-sorted structure which admits interpretations of modal formulae without nested modal operators.

In the second part of the contribution, we exploit the potential of step frames for investigating proof-theoretic aspects. This includes developing a method which detects when a specific rule-based calculus $A x$ axiomatizing a given logic $L$ has the so-called bounded proof property. This property is a kind of an analytic subformula property limiting the proof search space. We prove that every finite conservative step frame for $A x$ is a p-morphic image of a finite Kripke frame for $L$ iff $A x$ has the bounded proof property and $L$ has the finite model property. This result, combined with a 'step version' of the classical correspondence theory, turns out to be quite powerful in applications. For simple logics such as $\mathbf{K}, \mathbf{K} 4, \mathbf{S 4}$, etc, establishing basic matatheoretical properties becomes a completely automatic task (the related proof obbligations can be instantaneously discharged by current first-order provers). For more complicated logics, some ingenuity is still needed, however we were able to successfully apply our uniform method to Avron's cut-free system for GL and to Goré's cut-free system for S4.3.
\end{abstract}

\section{References}

[1] N. Bezhanishvili and S. Ghilardi. Bounded proofs and step frames. In Proc. of Tableaux 2013, Lecture Notes in Computer Science. Springer, 2013. Extended version available as a Technical Report at http://www.phil.uu.nl/preprints/lgps/number/306

[2] N. Bezhanishvili, S. Ghilardi, and M. Jibladze. Free modal algebras revisited: the step-by-step method. In Leo Esakia on Duality in Modal and Intuitionistic Logics, Trends in Logic. Springer, 2013. To appear.

[3] D. Coumans and S. van Gool. On generalizing free algebras for a functor. Journal of Logic and Computation, 23(3):645-672, 2013. 